 \\ www4.fsanet.com.br/revista
}

Rev. FSA, Teresina, v. 19, n. 1, art. 2, p. 24-38, jan. 2022

ISSN Impresso: 1806-6356 ISSN Eletrônico: 2317-2983

http://dx.doi.org/10.12819/2022.19.1.2

\section{A Legitimidade da Acreditação Hospitalar}

\section{The Legitimacy of Hospital Accreditation}

Tiago Martins Ramos da Silva

Mestre em Administração pela UNIGRANRIO Analista de Gestão em Saúde da Fundação Oswaldo Cruz

E-mail: tiagomartins.fiocruz@gmail.com

Clayton Pereira Gonçalves Doutor em Administração pela UNIGRANRIO Professor da Universidade Federal de Rondônia E-mail: clayton.goncalves@unir.br

Endereço: Tiago Martins Ramos da Silva

Fundação Oswaldo Cruz, Instituto Fernandes Figueira Avenida Rui Barbosa, 716, Flamengo, CEP: 22250020 , Rio de Janeiro, RJ - Brasil.

Endereço: Clayton Pereira Gonçalves

Universidade Federal de Rondônia - Avenida Rotary Club, 3756, Jardim Social, CEP:76981283, Vilhena, RO - Brasil.
Editor-Chefe: Dr. Tonny Kerley de Alencar Rodrigues

Artigo recebido em 21/07/2021. Última versão recebida em 10/08/2021. Aprovado em 11/08/2021.

Avaliado pelo sistema Triple Review: a) Desk Review pelo Editor-Chefe; e b) Double Blind Review (avaliação cega por dois avaliadores da área).

Revisão: Gramatical, Normativa e de Formatação 


\title{
RESUMO
}

Neste artigo, buscamos identificar a fonte de legitimidade que balizou a adoção da Acreditação Hospitalar em quatro hospitais do Rio de Janeiro, além de aferir se sua adoção se alinhava a questões de eficiência organizacional. Utilizando-se dos conceitos de Scott (1995) sobre tipos de legitimidade, realizamos uma pesquisa composta por entrevistas em profundidade e questionários semiestruturados com gestores e funcionários. O resultado mostrou que a legitimidade normativa melhor explicou a adoção da acreditação hospitalar em hospitais públicos e privados do Rio de Janeiro. As buscas por valores normativos socialmente aceitos, como qualidade do atendimento e segurança do paciente, determinaram os objetivos dos hospitais adotantes da acreditação hospitalar. A busca por eficiência também restou comprovada, evidenciando que mesmo em ambientes altamente institucionalizados, como no caso dos hospitais acreditados (Ruef e Scott, 1998), a eficiência pode ir de encontro à busca por legitimidade, destituindo as práticas de certificação hospitalar como meros mitos organizacionais.

PALAVRAS-CHAVE: Legitimidade. Acreditação Hospitalar. Hospitais. Organizações. Eficiência.

\begin{abstract}
In this article, we sought to identify the source of legitimacy that guided the adoption of Hospital Accreditation in four hospitals in Rio de Janeiro, in addition to assessing whether its adoption was aligned with issues of organizational efficiency. Using Scott's (1995) concepts about types of legitimacy, we conducted a survey consisting of in-depth interviews and semistructured questionnaires with managers and employees. The result showed normative legitimacy best explained the adoption of hospital accreditation in public and private hospitals in Rio de Janeiro. The search for socially accepted normative values, such as quality of care and patient safety, determined the goals of hospitals that adopted hospital accreditation. The search for efficiency was also proven, evidencing that even in highly institutionalized environments, as in the case of accredited hospitals (Ruef and Scott, 1998), efficiency can go against the search for legitimacy, dismissing hospital certification practices as mere organizational myths.
\end{abstract}

KEYWORDS: Legitimacy. Hospital Accreditation. Hospitals. Organizations. Efficiency. 


\section{INTRODUÇÃO}

Hospitais públicos e privados vêm passando por mudanças ao longo dos anos, visando principalmente a uma melhoria do atendimento à população e a consequente legitimidade de seus processos de trabalho. Em pesquisa realizada pela Datafolha, a pedido do Conselho Federal de Medicina em 2014, mais da metade dos entrevistados qualificou o sistema de saúde brasileiro com nota abaixo de 5 de um total de 10 pontos. Para os não usuários do SUS, o resultado foi ainda pior: $60 \%$ avaliaram a saúde no Brasil com nota abaixo de 5. Para Jorge et al. (2013), o Brasil sofre com aumento de gastos na saúde pública, além de problemas com o crescimento e o financiamento da rede privada de saúde. Esses fatores têm dificultado ainda mais a busca pela eficiência e qualidade dos serviços de saúde.

Visando a um aumento da eficiência e da qualidade nos serviços da saúde, um dos mecanismos que tem conquistado espaço dentro das organizações hospitalares são os certificados de qualidade chamados de acreditação hospitalar. Acreditação Hospitalar é um processo voluntário e não governamental realizado por organizações sem fins lucrativos, que avaliam as organizações hospitalares por meio de uma série de requisitos envolvendo aspectos de segurança do paciente e do funcionário, padrões técnicos, gerenciais, entre outros (ALONSO et al., 2014). Após a avaliação realizada pela organização acreditadora, o hospital adquire a certificação de hospital acreditado, caso consiga atingir os padrões exigidos pela mesma. Este trabalho considerou duas instituições acreditadoras em hospitais brasileiros: a Organização Nacional de Acreditação (ONA) e o Consórcio Brasileiro de Acreditação (CBA).

A adoção cada vez mais frequente desse processo de acreditação hospitalar em organizações de saúde brasileiras já motivou estudos acadêmicos sobre o assunto, como os trabalhos de Manzo et al. (2012), Jorge et al (2013) e Alonso et al. (2014). Entretanto, trabalhos buscando identificar a legitimidade dessas certificações ainda permanecem raros.

Este trabalho tem como objetivo identificar as razões que levaram as organizações hospitalares a aderirem ao processo de certificação hospitalar. Utilizando o trabalho de Scott (1995), buscamos analisar a fonte legitimadora da acreditação hospitalar, com foco na organização hospitalar adotante. Para alcançar o objetivo citado, realizamos entrevistas em profundidade e questionários semiestruturados com gestores e funcionários de quatro hospitais acreditados do Rio de Janeiro.

Após esta introdução, o conceito teórico de legitimidade é brevemente abordado. Em seguida, informações sobre o processo de acreditação hospitalar são explicitadas. $\mathrm{O}$ trabalho 
segue com as explicações metodológicas da pesquisa e, em seguida, os resultados são expostos. Uma conclusão com uma síntese dos achados finaliza a pesquisa.

\section{REFERÊNCIAL TEÓRICO}

\subsection{Legitimidade das Organizações}

Segundo Rossoni (2016), a utilização do conceito de legitimidade dentro do contexto organizacional tem início com Parsons $(1956,1960)$, ao situar organizações como imersas em um determinado ambiente social, que contém sistemas de valores que, consequentemente, são compartilhados. Quando o novo institucionalismo avança dentro dos estudos organizacionais, em meados dos anos 70, com trabalhos como o de Perrow (1974), Zucker (1977), entre outros, o estudo sobre legitimidade ganha notória importância, reanalisando o conceito sob os pilares de Weber (1991,2009). Para Rossoni (2016), dois motivos fundamentam essa revisitação: a impossibilidade de manipulação da legitimidade sob uma visão da teoria da dependência de recursos; e a agência da promoção da legitimidade por atores locais, ao invés de uma visão mais ampla do conceito, abordada principalmente pelo velho institucionalismo.

Mas o que é legitimidade? Diversos autores expuseram definições sobre o termo dentro da perspectiva neoinstitucionalista. Meyer e Rowan (1977), apesar de não apresentarem uma definição sobre legitimidade, ressaltaram sua importância para a sobrevivência das organizações. Meyer e Scott (1983) definem legitimidade como o grau de suporte cultural de uma organização. De acordo com Suchman (1995), legitimidade é uma percepção ou suposição generalizada de que as ações de uma entidade são desejáveis ou apropriadas dentro de uma estrutura de normas, valores, crenças e convicções socialmente construídas. Apesar do conceito de Schuman ser amplamente aceito no campo, ele ainda não define o que vem a ser uma organização legítima (ROSSONI, 2016). Segundo Rossoni (2016), "uma organização é considerada legítima quando seus elementos são suportados por princípios estruturais aceitos no sistema social". Em outras palavras, organizações legítimas são aquelas que possuem princípios e práticas que são amplamente aceitas pela sociedade na qual estão inseridas.

Para Scott (2008), a legitimidade não é simplesmente um recurso que pode ser comprado ou trocado, mas percebido, com ajuda de leis, regras, apoio externo e estruturas culturais-cognitivas. Diante da complexidade que envolve identificar a legitimidade nas práticas organizacionais, vários autores separaram-na em dimensões analíticas. Aldrich e Fiol 
(1994), Hunt e Aldrich (1996) classificaram a legitimidade em sociopolítica e cognitiva; Aldrich e Ruef (2006) acrescentaram uma terceira dimensão: aprendizagem; Archibald (2004), Stryker (1994) e Suchman (1995) criaram dimensões como Cultural, Moral e Pragmática. Apesar das diversas classificações existentes, todas elas tendem a seguir o modelo desenvolvido por Scott (1995), que distingue a legitimidade em regulatória, normativa e cultural-cognitiva (ROSSONI, 2016).

A legitimidade regulatória é aquela que se baseia em regras bem definidas. Organizações legítimas, segundo o pilar regulador, seguem regras definidas no respectivo campo social no qual estão inseridas (SCOTT, 2008; ROSSONI, 2016).

Já a legitimidade normativa compreende um sistema social normativo, composto por normas e valores. Organizações são legítimas quando seguem normas prescritas e valores socialmente aceitos e desejáveis (RUEF, SCOTT, 1998; SCOTT, 2008).

Por fim, a legitimidade cultural-cognitiva, que é o ponto central na distinção entre velho e neoinstitucionalismo, baseia-se em padrões culturais e cognitivos tomados como certos pela sociedade, de forma que a organização é considerada legítima se atende a esses anseios e posições na sociedade (RUEF, SCOTT, 1998; SCOTT, 2008).

A busca por legitimidade, entretanto, pode muitas vezes resultar em práticas que divergem dos demais objetivos organizacionais, sendo realizadas apenas cerimonialmente (MEYER; ROWAN, 1977). Meyer e Rowan (1977) argumentam que muitos mitos gerados por práticas organizacionais particulares e difundidos por redes relacionais têm legitimidade baseada na suposição de que são racionalmente efetivos. Entretanto, muitas práticas legítimas são copiadas por organizações, tornando-as isomórficas, sem necessariamente haver uma avaliação racional das suas consequências (DIMAGGIO; POWELL, 1983).

Segundo DiMaggio e Powell (1983), ações eram imitadas nas organizações por serem consideradas legítimas. Entretanto, a sua eficiência e eficácia já não eram mais questionadas, gerando decisões tomadas sem o amparo fidedigno da racionalidade. Ainda de acordo com os autores, quando práticas se tornam altamente institucionalizadas acabam funcionando como mitos, especialmente em organizações altamente institucionalizadas. Diante do exposto, será possível uma busca por legitimidade sem desviar dos objetivos principais da Organização?

\subsection{A Acreditação Hospitalar}

No campo da saúde, a busca por qualidade teve início nos Estados Unidos, nas primeiras décadas do século XX, e essa prática expandiu-se por todo o mundo (FELDMAN et 
$a l, 2005)$. A primeira avaliação de qualidade realizada em 1918 apontou que menos de $15 \%$ das organizações hospitalares americanas estavam dentro dos critérios previamente definidos como aceitáveis.

Os processos de avaliação foram sendo aprimorados até que em 1951 foi criada a primeira Comissão Conjunta de Acreditação dos Hospitais (CCAH) e foi formada pela união de diversas associações na área de saúde. Um ano depois, estava lançado o primeiro programa de acreditação chamado de Joint Comission on Acreditation of Hospitals (JCAH). Esse programa surgiu de uma iniciativa privada e voluntária, entretanto propiciou uma maior regulação por meio de leis e programas de fiscalização no campo da saúde nos Estados Unidos. Desde então, os padrões de qualidade exigidos estão cada vez mais elevados (FELDMAN et al, 2005; ALONSO et al, 2014).

No Brasil, a busca pela categorização dos hospitais, assim como avaliação e mensuração de padrões mínimos de qualidade tiveram origem e prerrogativa de Órgãos do Estado. Segundo Alonso et al. (2014), o primeiro trabalho voltado para a qualidade na organização hospitalar foi em 1935, quando foi elaborada uma ficha para avaliação da qualidade hospitalar para a extinta Comissão de Assistência Hospitalar do Ministério da Saúde.

Desde então, diversas iniciativas ocorreram, porém o avanço era moroso. Somente em 1989, quando a Organização Mundial da Saúde (OMS) deu significativa importância à acreditação para o desenvolvimento da qualidade na saúde na América Latina, esse processo ganhou força no país. Após esse fato, quatro Estados (São Paulo, Rio de Janeiro, Rio Grande do Sul e Paraná) alavancaram projetos e encontros entre associações na área de saúde, buscando desenvolver a ideia da acreditação no Brasil. Em 1998, é criado o CBA, representante brasileira da Joint Commission International.

Um ano depois, é a vez da ONA, organização tipicamente nacional, criada após o lançamento do manual brasileiro de acreditação. Desde então, as duas organizações citadas acima são as principais instituições acreditadoras no país. Abaixo, elaboramos um quadro para melhor visualizar o avanço da busca pela qualidade no campo da saúde:

\section{Quadro 1 - Cronologia do processo de acreditação hospitalar}

\begin{tabular}{|c|l|c|}
\hline Ano & \multicolumn{1}{|c|}{ Estados Unidos } & \multicolumn{1}{|c|}{ Brasil } \\
\hline 1918 & $\begin{array}{l}\text { Realizada Primeira Avaliação de Hospitais - de } \\
692 \text { hospitais, apenas 89 estão dentro dos } \\
\text { padrões estabelecidos. }\end{array}$ & \\
\hline
\end{tabular}




\begin{tabular}{|c|c|c|}
\hline 1924 & $\begin{array}{l}\text { Criação pelo Colégio Americano de Cirurgiões } \\
\text { do Programa de Padronização Hospitalar - } \\
\text { PPH. }\end{array}$ & \\
\hline 1935 & & $\begin{array}{l}\text { Elaboração de ficha para avaliação da qualidade } \\
\text { hospitalar para a extinta Comissão de Assistência } \\
\text { Hospitalar do Ministério da Saúde. }\end{array}$ \\
\hline 1951 & $\begin{array}{l}\text { Criada a primeira Comissão Conjunta de } \\
\text { Acreditação dos Hospitais, chamada de CCAH. }\end{array}$ & $\begin{array}{l}\text { 1o.Congresso Nacional do Capítulo Brasileiro do } \\
\text { Colégio Internacional de Cirurgiões realizado em } \\
\text { São Paulo, estabelecendo padrões para Centro } \\
\text { Cirúrgico. }\end{array}$ \\
\hline 1952 & $\begin{array}{l}\text { Lançado o primeiro programa de acreditação } \\
\text { chamado de Joint Comission on Acreditation of } \\
\text { Hospitals. }\end{array}$ & \\
\hline 1956 & & $\begin{array}{l}\text { Publicação do Decreto } 25465 \text { de } 1956 \\
\text { estabelecendo padrões para classificação de } \\
\text { Organizações de Saúde. }\end{array}$ \\
\hline 1970 & $\begin{array}{l}\text { Publicação do Accreditation Manual for } \\
\text { Hospital estabelecendo padrões de qualidade } \\
\text { mais elevados. }\end{array}$ & \\
\hline 1987 & $\begin{array}{l}\text { Nome da organização mudou de Joint } \\
\text { Comission on Acreditation of Hospitals } \\
\text { (JCAH) para Joint Commisson on } \\
\text { Accreditation of Healthcare Organization } \\
\text { (JCAHO) - com novas atividades inseridas, } \\
\text { ampliando o campo de atuação. }\end{array}$ & \\
\hline 1990 & & $\begin{array}{l}\text { Realizado um convênio com a Organização Pan- } \\
\text { Americana de Saúde - OPAS, a Federação } \\
\text { Latino-Americana de Hospitais e o Ministério da } \\
\text { Saúde para elaborar o Manual de Padrões de } \\
\text { Acreditação para América Latina. }\end{array}$ \\
\hline 1992 & & $\begin{array}{l}\text { Realizado o primeiro seminário nacional de } \\
\text { Acreditação em Brasília. }\end{array}$ \\
\hline 1994 & $\begin{array}{l}\text { Publicação o Comprehensive Accreditation } \\
\text { Manual for Hospitals - JCAHO desenvolve } \\
\text { padrões em nível internacional para aplicação } \\
\text { fora do país. }\end{array}$ & $\begin{array}{l}\text { Comissão Nacional de Qualidade e } \\
\text { Produtividade em Saúde - CNQPS lançada pelo } \\
\text { Ministério da Saúde. } \\
\text { Simpósio "Acreditação de Hospitais e Melhoria } \\
\text { de Qualidade em Saúde" com participação de } \\
\text { duas instituições acreditadoras internacionais } \\
\text { (EUA e Canadá). }\end{array}$ \\
\hline 1998 & & $\begin{array}{l}\text { Congresso Internacional de Qualidade na } \\
\text { Assistência à Saúde em Budapeste - Lançamento } \\
\text { do Programa Brasileiro de Acreditação. } \\
\text { Criação do Consórcio Brasileiro de Acreditação } \\
\text { - CBA. }\end{array}$ \\
\hline 1999 & & $\begin{array}{l}\text { Fundação da ONA - Organização Nacional de } \\
\text { Acreditação. }\end{array}$ \\
\hline
\end{tabular}

Fonte: Adaptado de Fieldman et al (2005 
A ONA define acreditação hospitalar como um sistema de avaliação e certificação da qualidade dos serviços de saúde, cujo caráter é eminentemente educativo, voluntário, sem finalidade fiscalizadora ou regulatória.

O processo de acreditação envolve toda a equipe da organização hospitalar, incluindo médicos, enfermeiros, técnicos, diretores, administradores, etc. As organizações avaliadoras seguem um rigoroso manual que contém uma série de protocolos que devem ser seguidos à risca para que a organização seja certificada. Enquanto a CBA certifica a organização hospitalar como acreditada ou não acreditada, a ONA as certifica em até três níveis: Nível 1: segurança; Nível 2: segurança e organização e Nível 3: segurança, organização e práticas de gestão de qualidade. Caso a organização não alcance o padrão mínimo do Nível 1, é considerada não acreditada (ONA, 2014; CBA, 2011).

Este processo de acreditação difere dos demais por ser de exclusividade da área da saúde, envolvendo não somente padrões mais fortes de ética e moralidade, mas também de aspectos mais técnicos e eficazes (ONA, 2014; CBA, 2011). Além do exposto, trata-se de um processo de alto custo financeiro, não obrigatório e sem incentivos fiscais por parte dos entes estatais.

Diante do exposto, qual o papel legitimador da prática de acreditação hospitalar, segundo os próprios adotantes? A acreditação hospitalar age de forma altamente institucionalizada e funciona como um mito objetivando apenas legitimidade?

Para responder a essas questões, utilizamos como base para análise os pilares legitimadores de Scott (1995), com a seguinte definição constitutiva e operacional de cada categoria:

Quadro 2 - Categorias analíticas

\begin{tabular}{|c|c|c|}
\hline Categoria & Definição Constitutiva & Definição Operacional \\
\hline Legitimidade Regulatória & $\begin{array}{l}\text { Nasce da conformidade de regras } \\
\text { explícitas emitidas por Órgãos } \\
\text { oficiais ou reguladores e } \\
\text { reconhecidas pelo sistema social } \\
\text { (Ruef; Scott, 1998; Scott, 1995, } \\
\text { 2008). Exemplo: Leis e } \\
\text { regulamentos que são seguidos e } \\
\text { reconhecidos. }\end{array}$ & $\begin{array}{l}\text { Hospitais que adotam a } \\
\text { Acreditação } \quad \text { Hospitalar para } \\
\text { cumprir determinada regra, lei ou } \\
\text { regulamento. }\end{array}$ \\
\hline Legitimidade Normativa & $\begin{array}{l}\text { Origina-se do reconhecimento pelo } \\
\text { ambiente no qual a organização } \\
\text { está inserida, de que determinada } \\
\text { ação alcança valores e normas } \\
\text { aceitos e desejáveis. }\end{array}$ & $\begin{array}{l}\text { Hospitais que adotam a } \\
\text { Acreditação Hospitalar para } \\
\text { satisfazer normas e valores de } \\
\text { clientes ou stakeholders dentro do } \\
\text { seu ambiente. }\end{array}$ \\
\hline Legitimidade Cultural-Cognitiva & Considerada aquela que é aceita & Hospitais que adotam a \\
\hline
\end{tabular}




\begin{tabular}{|l|l|l|}
\hline & $\begin{array}{l}\text { pelo ambiente de forma presumida; } \\
\text { uma prática tomada como certa e e } \\
\text { imediatamente reconhecida como } \\
\text { tal pelo sistema social. }\end{array}$ & $\begin{array}{l}\text { Acreditação Hospitalar por } \\
\text { entenderem ser uma prática } \\
\text { indispensável, sem margens para } \\
\text { questionamentos e dúvidas. É } \\
\text { tomada como certa. }\end{array}$ \\
\hline
\end{tabular}

Fonte: Adaptado de Scott (1995)

\section{METODOLOGIA}

Para realização desta pesquisa, optou-se pela estratégia de estudo qualitativo instantâneo (FLICK, 2008), onde são coletados, por meio de entrevistas, manifestações acerca do objeto de pesquisa e realizada uma comparação entre os achados, com o objetivo de explicar os fatos e circunstâncias no momento da pesquisa. Foram selecionados 4 hospitais localizados na cidade do Rio de Janeiro, sendo dois acreditados pela ONA e dois pelo CBA. A escolha dessas duas organizações acreditadoras é justificada pelo fato de ambas as certificadoras já estarem consolidadas no campo e possuírem um maior número de clientes. De acordo com dados extraídos dos sites de ONA e CBA, as duas certificadoras somadas possuem mais de 380 hospitais acreditados e, se consideradas as certificações setoriais, como ambulatórios, laboratórios, entre outros, os números ultrapassam 1500 certificações. Somente no Rio de Janeiro, mais de 30 hospitais possuem certificação de acreditação hospitalar válida.

O nível de análise da pesquisa é organizacional e as unidades de análise utilizadas foram os hospitais acreditados pela ONA ou CBA do Estado do Rio de Janeiro. Abaixo segue quadro informativo dos objetos de pesquisa analisados. O período de realização da pesquisa nas unidades de análise ocorreu entre julho/2015 e dezembro/2016.

Quadro 3 - Características dos objetos de pesquisa

\begin{tabular}{|c|c|c|c|c|}
\hline Hospital & Tempo de Fundação & $\begin{array}{l}\text { Personalidade } \\
\text { Jurídica }\end{array}$ & $\begin{array}{l}\text { Tempo de } \\
\text { Acreditação }\end{array}$ & $\begin{array}{l}\text { Organização } \\
\text { Acreditadora }\end{array}$ \\
\hline A & 20 ANOS & Privado & 14 ANOS & ONA \\
\hline B & 26 ANOS & Privado & 14 ANOS & ONA \\
\hline C & 39 ANOS & Público & 14 ANOS & CBA \\
\hline D & 72 ANOS & Público & 04 ANOS & CBA \\
\hline
\end{tabular}

A coleta de dados iniciou-se com a obtenção dos manuais ONA e CBA, dos quais foram extraídas informações sobre o processo de acreditação, envolvendo a solicitação pela organização hospitalar, formas de avaliação, bases e requisitos mínimos para obtenção do certificado. O manual da ONA utilizado foi a versão 2014, composto de 159 páginas, dividido em 5 seções que avaliam os hospitais de acordo com Gestão e Liderança, Atenção ao 
Paciente/Cliente, Diagnóstico e Terapêutica, Apoio Técnico e Abastecimento e Apoito Logístico. O manual da CBA utilizado foi a versão 2012 composto de 296 páginas, dividido em 2 seções: uma com padrões com foco no paciente e outra com padrões de administração de instituições de saúde. Os manuais de Acreditação Hospitalar das organizações acreditadoras ONA e CBA concentram todos os padrões que as organizações devem seguir para se tornarem acreditadas. Esta base de dados contribuiu para formular os questionários semiestruturados e as entrevistas em profundidade, uma vez que propiciou uma visão prática do procedimento de avaliação.

Em seguida, foram realizadas as entrevistas em profundidade com os avaliadores, sendo um de cada organização acreditadora, com o objetivo de enriquecer o conhecimento do processo de acreditação, assim como sanar dúvidas que existiram durante a leitura dos manuais.

Para a terceira etapa da pesquisa, foram selecionados 4 gestores, sendo um de cada hospital participante da pesquisa. Optou-se pelos gestores diretamente envolvidos com o processo de acreditação, geralmente lotados no setor de qualidade das organizações. Foi desenvolvido um roteiro prévio, com 16 questões norteadoras, envolvendo as categorias analíticas pesquisadas. Foram realizadas um total de 04 entrevistas em profundidade com os gestores de cada hospital selecionado, que foram gravadas e transcritas, resultando em um total de 04 horas e 12 minutos de áudio e 22 páginas de texto transcrito.

Após as entrevistas em profundidade, foram abordados 3 funcionários de cada hospital acreditado, por meio de questionários estruturados compostos de 11 questões, o que resultou em 59 páginas divididas em 12 funcionários, sendo na sua maioria enfermeiros e médicos, que estavam diretamente envolvidos com rotina de implantação da acreditação nos hospitais em que atuam.

A observação de campo ocorreu durante as entrevistas com os gestores, assim como durante a abordagem aos funcionários envolvidos, quando foi possível observar a rotina de trabalho da organização, assim como o funcionamento de alguns processos objetos de avaliação pelas organizações acreditadoras.

Com base no trabalho de Meyer e Rowan (1977), elaboramos dois tipos de objetivos pretendidos pela organização ao aderir ao processo de acreditação hospitalar: legitimidade, com o intuito de serem aceitas e respeitadas pelo ambiente, envolvendo clientes, fornecedores e concorrentes; eficiência, quando a organização tem como foco principal melhorar o aspecto técnico da atividade que exerce, como reduzir custos, melhorar procedimentos, aumentar a produtividade, etc. 
Para identificação desses objetivos foram realizadas perguntas principalmente aos gestores dos hospitais como: a). Quando a Instituição decidiu adotar a Acreditação Hospitalar no Hospital? b). O que você entende como Acreditação Hospitalar? Como a definiria? c). Por quais razões decidiu-se adotar a Acreditação Hospitalar? Tinham outras opções? d). Por que você considera a Acreditação Hospitalar importante para o Hospital? e) O que a instituição esperava alcançar com a adoção da Acreditação Hospitalar?

\section{RESULTADOS E DISCUSSÃO}

Ficou constatado que o processo de acreditação hospitalar, além de não ser obrigatório, também não fornece nenhum incentivo fiscal ou financeiro por parte do Estado e Órgãos reguladores para as organizações acreditadas. Além do exposto, trata-se de um processo que pode ser longo e oneroso, com custos maiores à medida que é necessário mais tempo para conclusão da certificação.

“O próprio processo de acreditação é bem caro, a gente teve que pagar agora mais de duzentos mil" (Gestor - Hospital C).

"Eu já vi um hospital que era certificado pela ISO já havia 8 anos (...) Quando eles entraram para o processo de acreditação já tinham uma documentação toda estruturada e tal. Mas ainda levaram quase 3 anos (...) eu vi uma instituição que levou 9 anos. Mas com muita dificuldade." (Avaliadora)

Nenhum dos entrevistados demonstrou um caráter regulatório na escolha da adoção. Todos os gestores indicaram objetivos mais relacionados a questões técnicas, como segurança do paciente e melhoria da qualidade dos processos, descartando a hipótese de uma legitimidade regulatória.

“A gente entende acreditação... é como uma ferramenta. Ela não é modelo. Modelo é modelo de qualidade, modelo de gestão. Modelo de gestão é um modelo de qualidade integrada e acreditação é uma ferramenta, acreditação não é modelo. Não é mesmo" (Gestor Hospital A). 
"Decidimos adotar para proporcionar um compromisso transparente de melhorar a segurança e qualidade do cuidado ao paciente" (Gestor Hospital B).

$\mathrm{O}$ aspecto cultural-cognitivo também foi pouco presente nas respostas. A maioria dos funcionários conhecia vagamente o processo de acreditação, enquanto os gestores reforçaram a necessidade de alcançar questões técnicas, porém não encararam a certificação como única opção, mas como uma escolha. Além do exposto, o processo de acreditação no Brasil ainda é muito recente, tornando difícil a institucionalização da prática diante do curto espaço de tempo desde as adoções iniciais.

"Nessa época (adoção) o Hospital já possuía uma caminhada de oito anos com outra certificação, a NBR ISO 9001, já com todos os processos mapeados e com registros das atividades, planos e políticas bem instituídas."

"Escolhermos a acreditação para obter uma maior qualidade no atendimento..." (Gestor hospital D).

Ficou evidenciado que o cliente do hospital não conhece a acreditação hospitalar. Os stakeholders também demonstraram exercer pouca influência na adoção, o que distanciou a questão do mimetismo (DiMaggio e Powell, 1983). Entretanto, todos os gestores reforçaram a necessidade de se obter valores normativos como mais qualidade e segurança no atendimento aos pacientes, destacando a legitimidade normativa frente a regulatória e cultural-cognitiva. Isso evidencia que as organizações hospitalares buscavam alcançar legitimidade, mas também eficiência, pois os clientes não enxergavam o selo de acreditação, mas as consequências trazidas por este. Foram identificados 34 trechos nas entrevistas que fazem referência aos objetivos da acreditação hospitalar relacionados à legitimidade ou eficiência. Dos 34 trechos encontrados, $80 \%$ estão relacionados com eficiência, enquanto os outros $20 \%$ com legitimidade.

"Eles (pacientes) não conhecem (Acreditação Hospitalar). Conseguem (pacientes) perceber a melhora, mas não por causa de uma ferramenta ou pelo selo de acreditação. Agora as pessoas vendem...ah depois que for acreditado vai trazer mais gente... traz ninguém" (...) é isso que te falei, por exemplo, se ele entrar aqui e ele tiver uma pulseirinha que tiver identificado e todo mundo olhar a pulseirinha e ele for no 
hospital que não usa, ele vai falar por que ele colocou e esse não? Por que quando tenho alergia um hospital botou e outro não? (Gestor Hospital A).

"Acho que (objetivo da acreditação) para manter uma maior organização e um maior cuidado com os pacientes. " (Funcionário - Hospital B)

"Muita. Muitas melhoras em diversas frentes. Mais a integração entre as equipes, na questão da educação do paciente, a gente realmente não prestava atenção nesse tipo de coisa" (Gestor - Hospital C)

\section{CONCLUSÃO}

Considerando que a acreditação hospitalar possui características peculiares e pouco exploradas, aferir a legitimidade de sua adesão pelas organizações de saúde foi o objetivo central deste trabalho.

Revisitando os conceitos legitimadores de Scott (1995), a legitimidade normativa melhor explicou a adoção da acreditação hospitalar em hospitais públicos e privados do Rio de Janeiro. As buscas por valores normativos socialmente aceitos, como qualidade do atendimento e segurança do paciente, determinaram os objetivos dos hospitais adotantes da acreditação hospitalar. Isso fica mais evidente quando observamos que o processo não é obrigatório, não oferece incentivos fiscais do Estado, além de ser recente dentro do campo de saúde brasileiro. Mesmo atrelado ao aspecto normativo, a busca por eficiência também restou comprovada, evidenciando que mesmo em ambientes altamente institucionalizados, como no caso dos hospitais acreditados (RUEF; SCOTT, 1998), a eficiência pode ir de encontro à busca por legitimidade, destituindo as práticas de certificação hospitalar como meros mitos organizacionais. É evidente que os achados desta pesquisa se limitam ao ambiente de saúde e à certificação de acreditação hospitalar. Como sugestão de trabalhos futuros, acreditamos que estudos envolvendo outras formas de certificação podem contribuir para o desenvolvimento da teoria sobre legitimidade das organizações, principalmente nas questões referentes ao cerimonialismo e à efetividade de tais práticas. 


\section{REFERÊNCIAS}

ALDRICH, H. E; FIOL, C. M. Fools rush in? The institutional context of industry creation. Academy of management review, v. 19, n. 4, p. 645-670. 1994.

ALDRICH, H. E; RUEF, M. Organizations evolving. (2006).

ALONSO, L. B. N et al. Acreditação hospitalar e a gestão da qualidade dos processos assistenciais. Perspectivas em Gestão \& Conhecimento, v. 4, n. 2, p. 34-49. 2014.

ARCHIBALD, M. E. Between isomorphism and market partitioning: How organizational competencies and resources foster cultural and sociopolitical legitimacy and promote organizational survival. In Legitimacy processes in organizations (pp. 171-211). Emerald Group Publishing Limited. 2004

DIMAGGIO, P. J; POWELL, W. W. The iron cage revisited: Institutional isomorphism and collective rationality in organizational fields. American sociological review, p.147-160. 1983

FELDMAN, L. B; GATTO, M. A.; CUNHA, I. C. K. O. História da evolução da qualidade hospitalar: dos padrões a acreditação. Acta paulista de enfermagem, v. 18, n. 2, p. 213-219. 2005

FLICK, U. (2008). Introdução à pesquisa qualitativa-3. Artmed editor. 2008.

HUNT, C. S; ALDRICH, H. E. Why even Rodney Dangerfield has a home page: Legitimizing the world wide web as a medium for commercial endeavors. In annual meeting of the Academy of Management, Cincinnati, OH. (1996, August).

JORGE, M. J; DE CARVALHO, F. A; DE OLIVEIRA MEDEIROS, R. Esforços de inovação organizacional e qualidade do serviço: um estudo empírico sobre unidades hospitalares. Revista de Administração Pública, v. 47, n. 2, p. 327-356. 2013.

MANZO, B. F; BRITO, M. J. M; CORRÊA, A. D. R. Implicações do processo de Acreditação Hospitalar no cotidiano de profissionais de saúde. Revista da Escola de Enfermagem da USP, v. 46, n. 2, p. 388-394. 2012.

MEYER, J. W. Centralization and the legitimacy problems of local government. Organizational environments: Ritual and rationality. 1983.

MEYER, J. W; ROWAN, B. Institutionalized organizations: Formal structure as myth and ceremony. American journal of sociology, v. 83, n. 2, p. 340-363. 1977.

PARSONS, T. Suggestions for a Sociological Approach to the Theory of Organizations-I. Administrative science quarterly, v. 1, n. 1). 1956.

PARSONS, T. Structure and process in modern societies. United State, University of Michigan. 1960.

PERROW, C. Organizational analysis: A sociological view London. 1974. 
ROSSONI, L. O que é legitimidade organizacional?. Organizações \& Sociedade, v. 23, n. 76), p110-129. 2016

RUEF, M; SCOTT, W. R. (1998). A multidimensional model of organizational legitimacy: Hospital survival in changing institutional environments. Administrative science quarterly, $\mathrm{p}$. 877-904. 1998

SCOTT, W. R. Institutions and organizations: Ideas and interests. Sage. 2008.

SCOTT, W. R. Institutions and Organizations. Thousand Oak. Cal: Sage Publications. 1995

STRYKER, R. Rules, resources, and legitimacy processes: Some implications for social conflict, order, and change. American Journal of Sociology, v. 99, n. 4, p. 847-910.1994

SUCHMAN, M. C. Managing legitimacy: Strategic and institutional approaches. Academy of management review, v. 20, n. 3, p. 571-610. 1995

WEBER, M. Economia e Sociedade. vol. 1. 5. ed. Brasília: UNB, 1991.

WEBER, M. The theory of social and economic organization. Simon and Schuster. 2009

ZUCKER, L. G. The role of institutionalization in cultural persistence. American sociological review, p. 726-743. 1977

Como Referenciar este Artigo, conforme ABNT:

SILVA, T. M. R; GONÇALVES, C. P. A Legitimidade da Acreditação Hospitalar. Rev. FSA, Teresina, v.19, n. 1, art. 2, p. 24-38, jan. 2022.

\begin{tabular}{|l|c|c|}
\hline \multicolumn{1}{|c|}{ Contribuição dos Autores } & $\begin{array}{c}\text { T. M. R. } \\
\text { Silva }\end{array}$ & $\begin{array}{c}\text { C. P. } \\
\text { Gonçalves }\end{array}$ \\
\hline 1) concepção e planejamento. & $\mathrm{X}$ & \\
\hline 2) análise e interpretação dos dados. & $\mathrm{X}$ & \\
\hline 3) elaboração do rascunho ou na revisão crítica do conteúdo. & $\mathrm{X}$ & $\mathrm{X}$ \\
\hline 4) participação na aprovação da versão final do manuscrito. & $\mathrm{X}$ & $\mathrm{X}$ \\
\hline
\end{tabular}

